\title{
Identifying Evolutionary Patterns of SMBHs Using Characteristic Variables of the Quasar AGNs of eBOSS
}

\author{
Sarah Martens \\ Department of Astronomy, University of Wisconsin- Madison
}

\begin{abstract}
We intend to investigate the redshift distribution and environmental conditions of quasar AGNs. We employ specific redshift bins within eBOSS to draw conclusions on the evolutionary path of supermassive black holes (SMBHs) and how it is dependent on environment; in addition we seek to determine the extent to which AGN feedback is responsible for heating within the intergalactic medium of galaxy groups.
\end{abstract}

\section{Introduction}

Sloan Digital Sky Survey's wide range of redshifts and extensive amount of targets including galaxies, quasars, and stars provides an excellent source from which to study evolutionary patterns of astrophysical bodies. We know the standard model of galaxy evolution: moving from young blue star forming galaxies to older red inactive galaxies, creating what is known as the blue cloud and the red sequence (Schawinski et al.2014). Features such as photometric colors can be analyzed to create an image of stellar population in galaxies with respect to their HI mass, (two values known to be inversely proportional- as gas is the fuel to produce stars). The widely accepted origins of active galactic nuclei are galaxy mergers during eras of structure developing (Lietzen, $\mathrm{H}$ et al.2016). These mergers trigger disruption of gas which can trigger a period of rapid star formation in galaxies, this gas also contributes to a large amount of infall which serves as fuel for an accreting black hole (Lietzen, $\mathrm{H}$ et al.2016, Hopkins, Philip F. et al.2005a). However, this rapid accretion will result in feedback and the expulsion of matter out of the black hole in the form of a quasar (Hopkins, Philip F. et al.2005a). After the strong quasar feedback clears the surrounding gas, we are able to visibly detect the quasar until the rate of accretion slows and the quasar turns off and slows the further growth of the black hole (Lietzen, $\mathrm{H}$ et al. 2016, Hopkins, Philip F. et al.2005a). However, the optical wavelengths aren't the only indicators of QSO characteristics, some QSOs can also be detected in the radio wavelength and are evidence for highly energetic activity, namely supermassive black holes. Environmental circumstances can have control over the evolutionary cycle of QSOs. For example, nearby QSOs tend to be located more towards the outer reaches of their respective groups and their evolution is very clearly connected to environmental densities and the large scale universal density (Lietzen, $\mathrm{H}$ et al.2016).

In this study we view the eBOSS DR13 QSO sample through multiple different lenses, by coupling the survey with other data catalogs in order to maintain the most well rounded view possible of the environments of our sample and the factors that may be affecting evolutionary patterns. We begin by exploring the Faint Images of the Radio Sky at Twenty-Centimeters (FIRST) catalog which we continue to work with and incorporate into our analysis. In order to gain insight into the environments present in the eBOSS QSO sample, particularly at low redshifts, we include the galaxy group catalog from Tempel, E. et al. 2012. We use distance from 
the QSO to the group center in degrees of RA and DEC and Z, within a set limit, to identify which QSOs are in groups, and eventually aim to return to the FIRST data to see in what environments they are most energetic and large. Combining our eBOSS data with the FIRST and Tempel catalogs by way of cross matching RA, DEC, and redshift measurements provides the most complete picture of the evolution of QSOs. In addition we intend to include an analysis of the colors (particularly R and G-R) of both the eBOSS QSO and galaxy samples to observe the star formation patterns and evolutionary phases of each and how it relates to the other factors of redshift, radio detections, and group membership.

\section{Data}

2a. eBOSS: The Extended Baryon Oscillation Spectroscopic Survey, or eBOSS, is a cosmological survey from SDSS-IV which extends the redshift limits of the BOSS survey and increases the amount of galaxies and QSOs detected, using baryonic acoustic oscillations (BAO) as a geometric probe. EBOSS focuses on ELGs (emission line galaxies) and QSOs (quasars) focusing primarily on the low redshift range between $0.6<\mathrm{z}<2.5$, although covering redshifts up to $\mathrm{z} \approx 7$. Along with the increased redshift range, and the amount of galaxies and QSOs detected, eBOSS yields more accurate Lyman $\alpha$ forest measurements. The Lyman $\alpha$ forest causes redshifting in the light from quasars and results in additional noisy features in quasar spectra as the light passes through dust in the intergalactic medium. The Lyman $\alpha$ forest is useful to those who are interested in the intergalactic medium, however when targeting quasar spectra it is something that scientist aim to eliminate. General goals of the eBOSS survey are to more accurately calibrate photometric redshifts in situations of weak lensing (lensing occurs when light is refracted due to an object acting as a lens between the observer and target), provide insight into cluster mass by obtaining precise redshifts and velocity dispersions of galaxies, and to produce a map of the grand universal structure in which the BAO feature is found.

The SDSS DR13 eBOSS data used in this research was obtained via the Sloan Digital Sky Survey Wiki and data access page (see Figure 1 for a general map of the sample). By downloading the large fits file and isolating the QSO sample using the python terminal, our working data set for this project was obtained. We work with a sample of 595025 QSOs ranging from redshift of $\mathrm{z}=0.6$ to $\mathrm{z} \approx 7$, spanning the entire range of the eBOSS survey (see Figure 4 for more on the redshift distribution of the sample). 


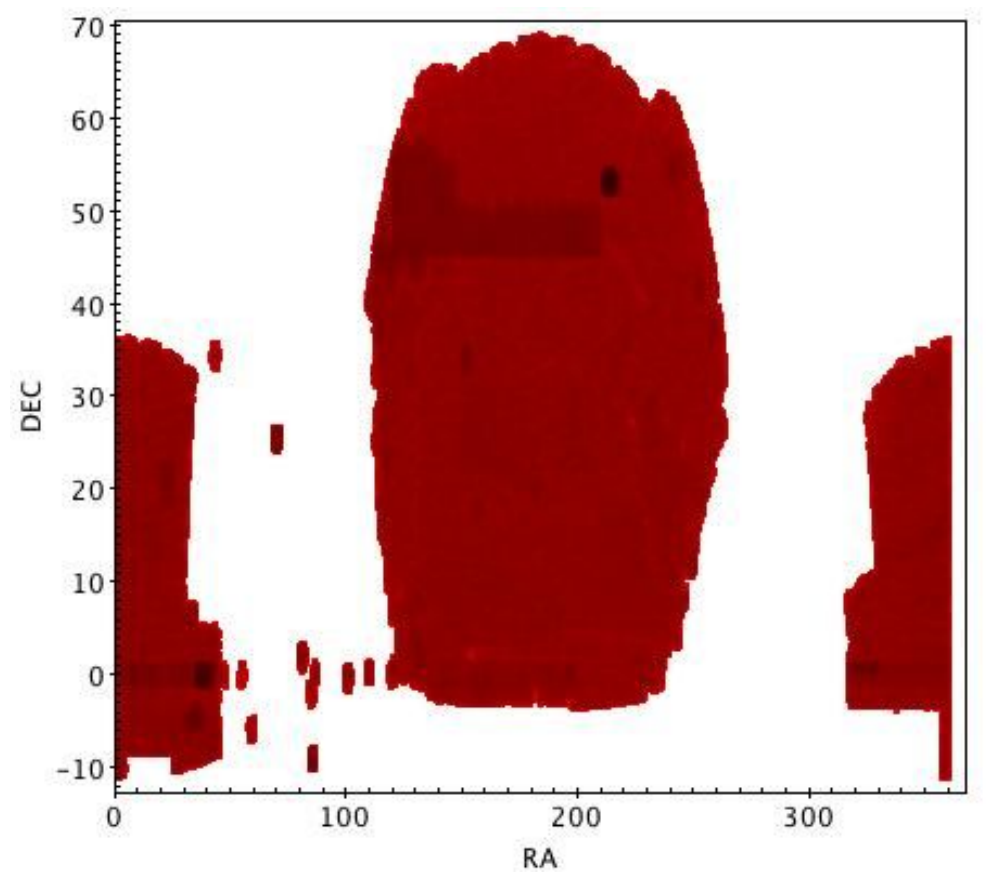

Figure 1: An RA and DEC map of the eBOSS DR13 sample (same for both the QSO and galaxy portions)

2b. FIRST: The Faint Images of the Radio Sky at Twenty-Centimeters, or FIRST, catalog is a radio catalog produced with data from the NRAO with the Jansky Very Large Array (VLA) in Socorro, NM. The goal of the FIRST survey is to produce a catalog similar to that of the Palomar Observatory Sky Survey while also covering a 10,000 square degree area on the sky (http://sundog.stsci.edu/first/description.html). Each hexagon grid exposure is 3 minutes per target and uses $2 \times 7$ 3-MHz frequency channels (http://sundog.stsci.edu/first/description.html). The FIRST information page states that about $15 \%$ of their radio sample has a corresponding optical detection in the Palomar Observatory Sky Survey (POSS) from Sloan Digital Sky Survey, as they chose their survey area specifically within that of SDSS (see Figures 1 and 2 to compare the coverage of eBOSS with the FIRST catalog). 


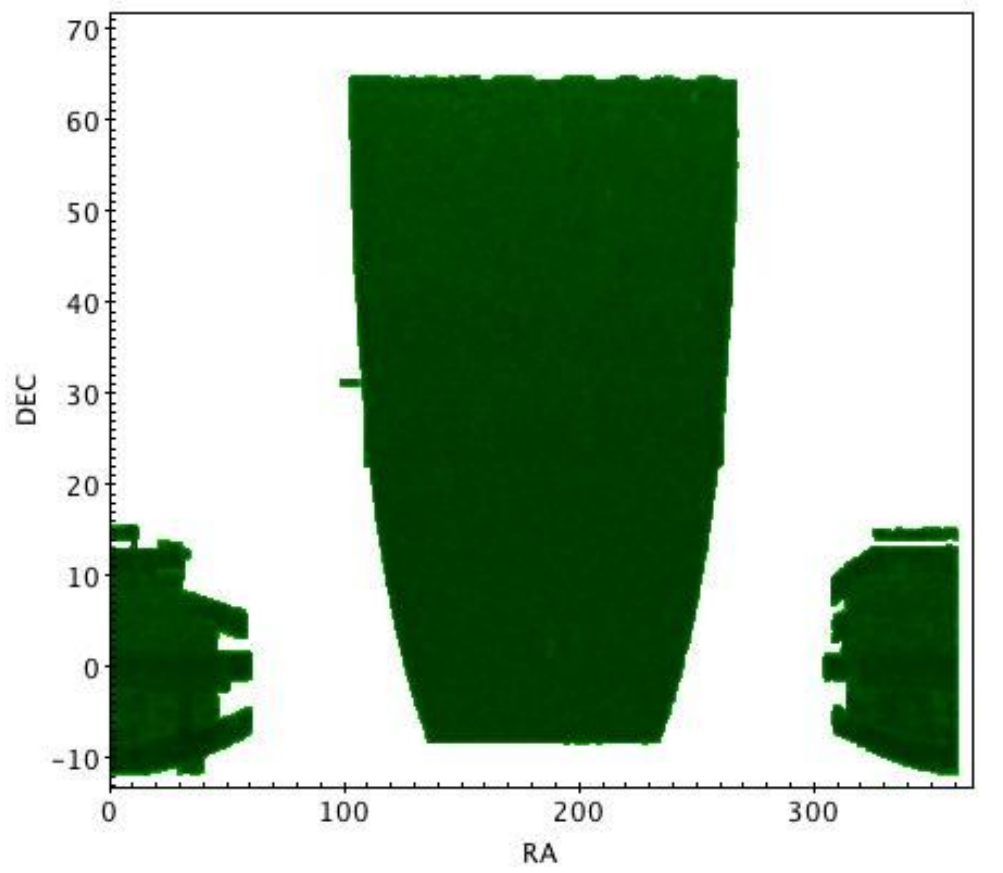

Figure 2: An RA and DEC map of the FIRST catalog.

2c. Tempel et. al. groups: Over half of all galaxies exist in groups, the environment most conducive to the evolution of the Universe. This makes galaxy groups a vital subject of study when considering the the large and small (global and local) scale structures of the universe (Tempel, E. et al.2012). The large scale structure is the very thing SDSS and eBOSS aim at doing in their large galaxy surveys, making galaxy group and clustering an easy and important topic to pair with SDSS data (see Figures 1 and 3 to compare the coverage of eBOSS and Tempel et al. 2012). After creating galaxy group catalogs from DR5 and DR7, and now with DR8, Tempel, E. et al. employs many strategies in order to produce a catalog of as many galaxy groups as possible. To optimize their results they use an altered friends-of-friends algorithm with a specifically tuned linking length, and additional environmental and morphological constraints, while also filling galaxy detections that are missing in SDSS due to fibre collisions (Tempel, E. et al.2012). Using $\mathrm{r}$ band luminosity, Tempel, E. et al. produce environmental densities for each galaxy they consider. Identifying these densities is helpful when studying the role of environment on the evolutionary processes affecting galaxies. Tempel, E. et al. also strengthens the morphology classifications made by Galaxy Zoo and have had their results confirmed by other science groups who ran similar tests using Galaxy Zoo (such as Huertas-Company et al. $2011 \mathrm{http}: / /$ www.aanda.org/articles/aa/pdf/2012/04/aa17632-11.pdf). For more on the details of the construction of their catalog see Tempel, E et al. 2012. 


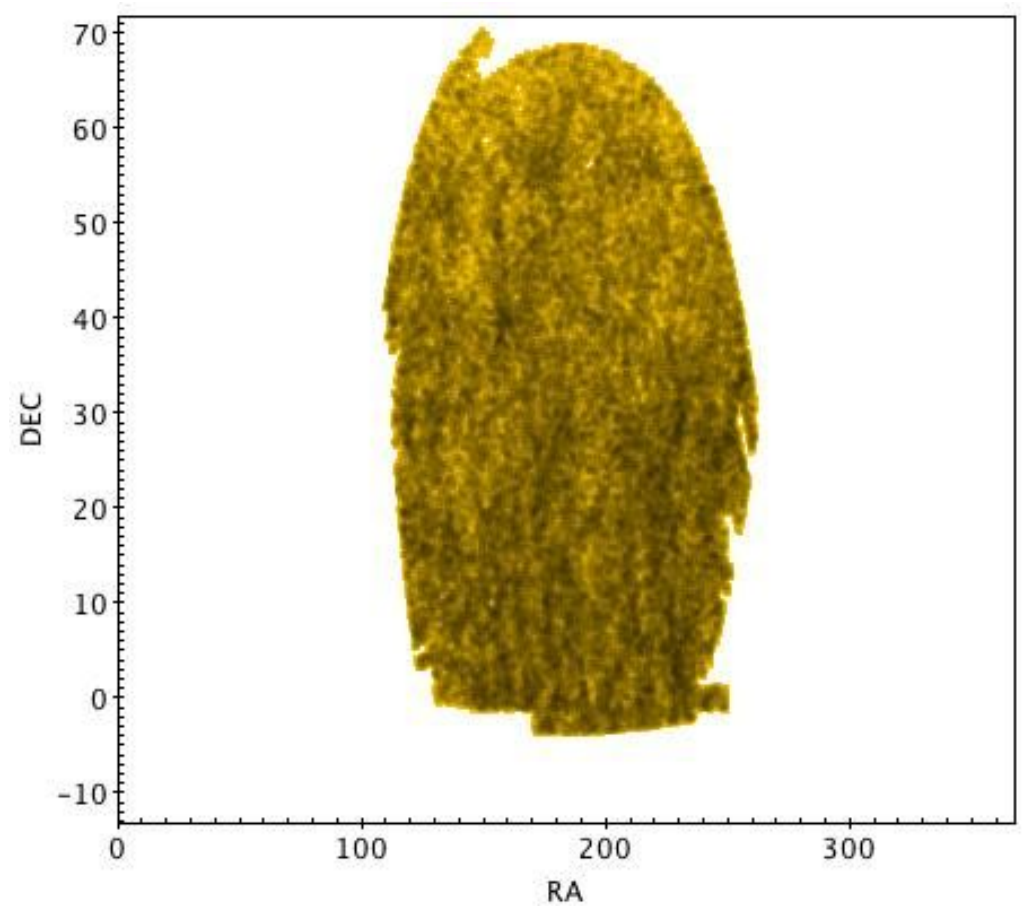

Figure 3: An RA and DEC map of the Tempel groups catalog.

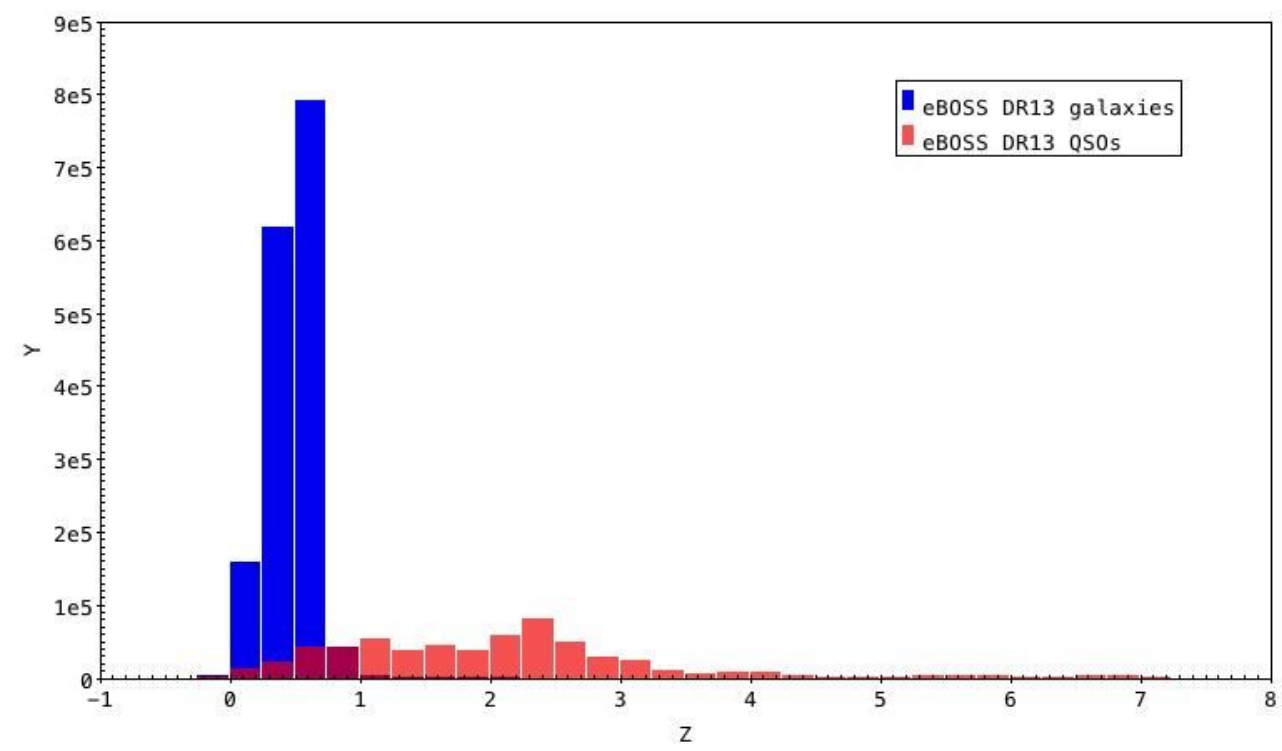

Figure 4: A histogram showing the redshift distribution of the eBOSS DR13 QSO sample (red), and how it compares to the redshift distribution of the the eBOSS DR13 galaxy sample (blue). The highly populated low redshift galaxies of DR13 allow us ready comparison in environment and color, however because the galaxy sample is limit in its redshift range we have to employ other sources at higher $\mathrm{z}$. 


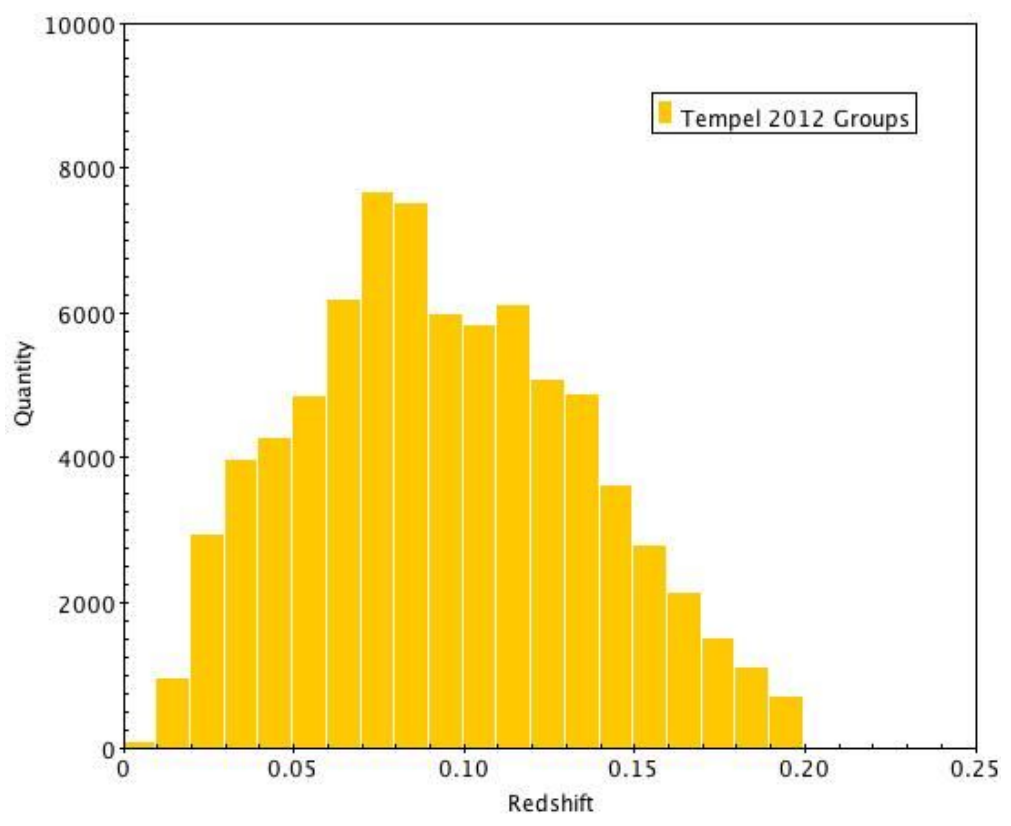

Figure 5: A histogram showing the redshift distribution of the Tempel, E. et al. groups over it's redshift range of $[0.009,0.2]$. The concentration of groups peaks between redshift 0.05 and 0.15 but the sample is generally well distributed over the range of the sample.

Data Catalogs and Samples Used

\begin{tabular}{|l|l|l|}
\hline Data Source & No. Rows & No. Matching Rows \\
\hline eBOSS DR13 QSOs & 595,025 & 595,025 \\
\hline eBOSS DR13 Galaxies & $1,618,808$ & 0 \\
\hline FIRST & 946,432 & Pending \\
\hline Tempel et. al Groups & 77,858 & 8,371 \\
\hline
\end{tabular}

Figure 6: Showing all of varieties of data used in this research, how many items (or rows) are included in each catalog and how many of those items are also detected in the eBOSS DR13 QSO sample-the focus of this research.

\section{Methodology}

We began this project by downloading the spALL-v5_9_0.fits file, containing the entire eBOSS DR13 from the eBOSS dedicated data access page on the SDSS wiki (http://trac.sdss.org). In order to read this large file, we installed the fitsio python package by Erin Sheldon before removing the QSO and galaxy portions of the sample into two new separate fits files. We then created the RA and DEC position plot seen in Figure 1 along with the histogram that can be seen in Figure 4, both using Tool for OPerations on Catalogues And Tables (TOPCAT). We then began analyzing the environment of the QSOs by creating another redshift histogram of the galaxy sample to compare the redshift locations of the groups. However, most of the galaxies in the eBOSS DR13 sample are at $\mathrm{z}<1$. This can provide useful insight into the environment of the low-z QSO sample, but not for the high-z range. Assessing the location of galaxy groups and 
clusters also posed a viable way to asses environment, so we made another redshift histogram (again using TOPCAT) of the galaxy groups defined in the Tempel, E. et al.2012 paper with a redshift range of $0.009<\mathrm{z}<0.2$. This overlaps with the low-redshift range the eBOSS QSOs sample, as can be seen in Figure 5. 1.61\% of the eBOSS QSO sample is within this range and we chose to investigate further via python in the Aquamacs interface. We cross matched the RA and DEC coordinates of the complete QSO sample with that of the galaxy groups from the Tempel, E. et al.2012 sample in order to see which of our QSOs existed within group environments (RA and DEC of the Tempel, E. et al. 2012 groups is measured from the center). Because we were comparing the RA and DEC of an individual point on the sky to the center of a group, more analysis was required in deciding the acceptable range of distance in which a QSO would be considered a member of the group in question. We converted each QSO redshift to a distance in megaparsecs using $D=\frac{c \times Z_{Q}}{70}$ and $A=\frac{D \times 3600}{206265}$ where $Z_{Q}$ is the redshift of the QSO, $c$ is the speed of light, and $A$ is the acceptable difference in distance from the group center. After calculating the distance of the QSOs from the group (using $B=\sqrt{\left(R A_{Q}-\operatorname{racl}_{T}\right)^{2}+\left(D E C_{Q}-\operatorname{deccl}_{T}\right)^{2}}$ where $R A_{Q}$ and $D E C_{Q}$ are the RA and DEC of the QSO and $\mathrm{racl}_{T}$ and $\mathrm{deccl}_{T}$ are the RA and DEC of the group center), we called for $\mathrm{A}>\mathrm{B}$ and for $\left|Z_{Q}-z c l\right| \leq 0.001$ for the QSO to be considered a member of a Tempel, E. et al.2012 group. This python code produced a file with a list of all of the QSOs detected in groups. This provided data that enabled a study of environment, focusing in the short term on those in the low redshift Tempel range.

We are currently in the process of cross referencing the large FIRST survey file with our eBOSS QSOs in a similar way as the Tempel groups. Once we can cross reference with FIRST catalog we will require that $\left|R A_{Q}-R A_{F}\right| \leq 0.001$ and $\left|D E C_{Q}-D E C_{F}\right| \leq 0.001$ (where $R A_{F}$ and $D E C_{F}$ are the RA and DEC measurements of a given FIRST galaxy) for the QSO to be consider radio detected, and therefore with a SMBH host. We now know that we have a significant amount of matches, but not yet a number that is confidently quantifiable. Pursuing this inclusion of FIRST will allow us to continue the work established in the previous section, and better our image of QSOs.

\section{Results}

4a. Groups and Clusters: In order to analyze the environment of our QSO sample, we employed the group and cluster catalog produced by Tempel et al. 2012 to cross reference our sample. The Tempel sample ranges from $[0.009,0.2]$ identifying low redshift groups and clusters with membership minimum of 2 , which is also the membership of the majority of their groups. Of our QSO sample, 9575 (1.61\% of our full sample) fell into the low redshift Tempel range, making it a good representation for only a small range of our sample. However, upon cross reference of the RA, DEC, and Redshift, we found that of the 9575: 87\% (8371 QSOS) are members of one of the groups from the Tempel catalog. The existence of AGNs is more probable in surrounding areas which are conducive to their growth, namely underdense areas where there are less processes pulling gas away from galaxies, and more importantly away from the black holes. (Silverman, J. D. et al. 2016). Because of this, quasars avoid rich superclusters and small 
groups (low density and low velocity dispersion) of galaxies are a favored environment for black holes (Hopkins et al., 2005b). This is consistent with our findings and the percent of matches we had with the majority of Tempel groups having very small membership (Lietzen, $\mathrm{H}$ et al. 2016, Tempel et al. 2012).

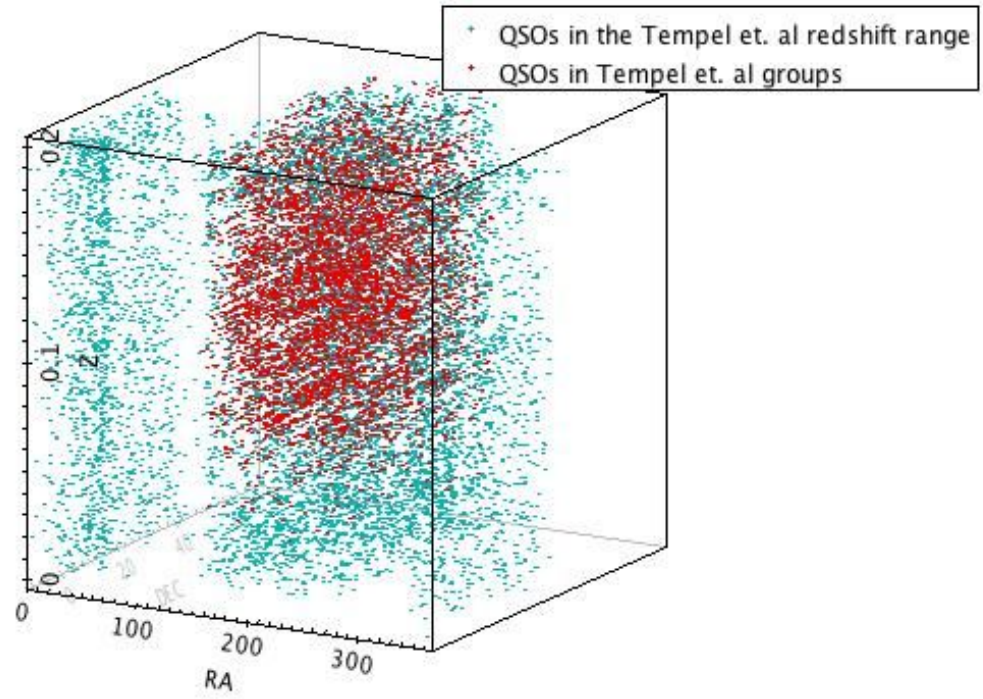

Figure 7: A 3D map of the QSOs within the Tempel redshift range $[0.009,0.2]$ in blue, and those QSO that were found to be part of the Tempel group catalog in red. The eBOSS RA and DEC range is larger than that of Tempel, E. et al. so the QSOs in groups are focused in the center of the 3D image, where the Tempel survey is present.

4b. FIRST: X-ray detections of optical QSOs are linked to galaxies currently accreting matter onto a SMBH (Silverman, J. D. et al.2016). We use the Faint Images of the Radio Sky at Twenty-Centimeters (FIRST) from the VLA as a radio source for our eBOSS sample in which to identify supermassive black holes. We obtained the FIRST catalog data from their website (http://sundog.stsci.edu/cgi-bin/searchfirst). We then used FIRST radio catalog $(1.4 \mathrm{GHz}$ ) as a cross reference with the RA and DEC of our QSO sample, to attempt to identify QSOs detected in the radio wavelengths. As stated above in Section 3, we choose 0.0001 degrees as our threshold of acceptable variance between the RA and DEC of a FIRST detection and any of our QSO sample. If the absolute value of the difference in both RA and DEC is less than 0.0001 we consider the our QSO to be the same point which is detected in the radio wavelengths, and therefore also identifying it as a Supermassive Black Hole due to its highly energetic nature and the range of wavelengths it spans (radio to optical). At this moment we have a number of eBOSS DR13 QSOs detected in the radio wavelengths.

\section{Conclusions}

In general $50-70 \%$ of galaxies are in group environments (Huchra and Geller 1982; Eke et al. 2004). We found that the population of low redshift QSOs in group environments is higher, at 
$87 \%$. This result is not shocking, as these groups tend to be lower density environments which will trigger accretion and activity in a QSO without stripping all of the gas, or fuel, from the black hole. As previously stated, QSOs detected in the radio $(1.4 \mathrm{GHz})$ in the FIRST catalog are likely SMBHs as only the most energetic QSOs will radiate in radio wavelengths. Along with the future work discussed above, we aim to continue assessing the different environmental conditions and evolutionary patterns of our QSO sample by quantifying the amount of eBOSS DR13 QSOs detected in the FIRST catalog and using R vs G-R color plotting in redshift bins. This color analysis will be a very important part of this project as it continues to progress but so far has been a task which has proven challenging for us. EBOSS DR13 houses a 5 element array containing the ugriz filter colors in units of nanomaggies. These are the colors with which we desire to make our color diagram (R vs G-R), however the units are not desirable when creating this visual and we seek a more streamlined way to convert them to the standard magnitude units.

In general, high mass AGNs tend to exist in less dense environments and have colors which are bluer, linking them with current star formation, than the general galaxy population (Silverman, J. D. et al.2016). A consideration of color will allow us to compare past results with the new DR13, along with viewing and analyzing the red sequence, green valley, and blue cloud phases of galaxy evolution. This will give us a better sense of the ages and star forming habits of both our QSO and galaxy data from eBOSS. Due to the large quantity of data in our samples we intend to use binning as a way to make our figures more manageable and readable. We plan on bins of one tenth at every integer $(\mathrm{z}=0-7)$ of redshift in our sample $(0.1-0.2,1.1-1.2,2.1-2.2 \ldots$ etc $)$.

These future endeavours may enhance the strength and variety of lenses through which we can view our data by: helping us gain insight into stellar age and phase, strengthening the visuals we provide in our analysis, and finding methods for measuring environment of high redshift QSOs.

\section{Acknowledgments}

I gratefully acknowledge Wisconsin Space Grant Consortium Undergraduate Research Grant and the Sloan Digital Sky Survey Collaboration for providing data and funding for this summer research.

\section{References}

[Dawson, Kyle S. et al., 2016] The Sdss-Iv Extended Baryon Oscillation Spectroscopic Survey: Overview And Early Data. The Astronomical Journal https://arxiv.org/abs/1508.04473

[Eke, V. R. et al., 2004] Galaxy Groups in the 2dFGRS: The Group-finding Algorithm and the 2PIGG Catalogue. Monthly Notices of the Royal Astronomical Society

https://arxiv.org/abs/astro-ph/0402567

[Hopkins, Philip F. et al., 2005a] A Physical Model for the Origin of Quasar Lifetimes. The Astrophysical Journal https://arxiv.org/abs/astro-ph/0502241 
[Hopkins, Philip F. et al., 2005b] Black Holes in Galaxy Mergers: Evolution of Quasars. The Astrophysical Journal https://arxiv.org/abs/astro-ph/0504190

[Huchra, J.P., and M.J. Geller, 1982] Groups of Galaxies. I - Nearby Groups. The Astrophysical Journal http://adsabs.harvard.edu/full/1982ApJ...257..423H

[Kneib, J.-P. et al.] E-BOSS (extended BOSS) The Novel Sloan Legacy Cosmological Survey. PDF of paper

https://indico.in2p3.fr/event/11823/attachments/5729/7135/eboss_2011_arc_proposal.pdf

[Lietzen, H et al., 2009] Environments of Nearby Quasars in Sloan Digital Sky Survey. Astronomy and Astrophysics https://arxiv.org/pdf/0904.0558.pdf

[Schawinski, K et al., 2014] The Green Valley Is a Red Herring: Galaxy Zoo Reveals Two Evolutionary Pathways towards Quenching of Star Formation in Early and Late-type Galaxies. Monthly Notices of the Royal Astronomical Society http://arxiv.org/abs/1402.4814

[Sheldon, Erin.] A Python Package for FITS Input/output Wrapping Cfitsio. GitHub.

[Silverman, J. D. et al.,2009] The Environments of Active Galactic Nuclei within the ZCOSMOS Density Field. The Astrophysical Journal https://arxiv.org/pdf/0812.3402.pdf

[Tempel, E., E. Tago, and L. J. Liivamägi., 2012] Groups and Clusters of Galaxies in the SDSS DR8. Astronomy amp; Astrophysics A amp Accessed data sample from:

http://www.aai.ee/ elmo/dr8groups/

[White, Richard L.] FIRST Catalog Search. 